\title{
T-odd generalized and quasi transverse momentum dependent parton distribution in a scalar spectator model
}

\author{
Xuan Luo, Hao Sun ${ }^{\text {a }}$ \\ Institute of Theoretical Physics, School of Physics, Dalian University of Technology, No. 2 Linggong Road, Dalian, Liaoning 116024, People's \\ Republic of China
}

Received: 26 May 2020 / Accepted: 25 August 2020 / Published online: 5 September 2020

(C) The Author(s) 2020

\begin{abstract}
Generalized transverse momentum dependent parton distributions (GTMDs), as mother funtions of transverse momentum dependent parton distributions (TMDs) and generalized parton distributions (GPDs), encode the most general parton structure of hadrons. We calculate four twisttwo time reversal odd GTMDs of pion in a scalar spectator model. We study the dependence of GTMDs on the longitudinal momentum fraction $x$ carried by the active quark and the transverse momentum $\left|\vec{k}_{T}\right|$ for different values of skewness $\xi$ defined as the longitudinal momentum transferred to the pion as well as the total momentum $\left|\vec{\Delta}_{T}\right|$ transferred to the pion. In addition, the quasi-TMDs and quasi-GPDs of pion have also been investigated in this paper.
\end{abstract}

\section{Introduction}

Exploring the partonic substructure of hadrons is still at the frontier of hadronic high energy physics research. The parton distribution functions (PDFs) make it clear how the longitudinal parton momentums in hadron are distributed. However, they only include one dimension information. Therefore probing how partons distribute in the transverse plane in both momentum and coordinate space becomes a vital topic. Typically, the transverse spatial distribution of partons inside a hadron can be quantified by generalised parton distributions (GPDs) [1-3], which can be denoted as a function of longitudinal momentum fraction $x$ carried by the parton, the longitudinal momentum $\xi$ transferred to the hadron and the total momentum transferred $t$. They can be accessed through measurements in hard exclusive reactions like deep virtual Compton scattering and hard exclusive meson production $[2,4]$. While the transverse momentum dependent parton distributions (TMDs) [5-7] depending on both the longitu-

\footnotetext{
ae-mail: haosun@mail.ustc.edu.cn; haosun@dlut.edu.cn (corresponding author)
}

dinal and transverse motion of partons inside a hadron can be studied by the description of various hard semi-inclusive reactions. More general distibutions than the GPDs and the TMDs, the generalized transverse momentum dependent parton distributions (GTMDs) [8-10] could reduce to them in specific kinematical limits, therefore serve as mother distributions. The GTMDs can directly enter the description of hard exclusive reactions. The parametrization of the generalized quark-quark correlation functions for a spin- 0 and spin- $1 / 2$ hadron in terms of GTMDs are given in Refs. [8,9]. Then the authors in Ref. [10] add a complete classification of gluon GTMDs. Particularly, the correlator related to the time-reversal odd (T-odd) GTMDs is contributed by the final state interactions from gauge link or Wilson line. These interactions are necessary to generate the single spin asymmetries [11].

Although PDFs are related to parton fields in QCD, it is difficult to calculate them directly in QCD since they are nonperturbative quantities. This difficulties may be overcomed by Lattice QCD method studying the PDFs from first principles. However, PDFs are usually defined on the light cone, which poses a problem for the standard Euclidean formulation, and in lattice QCD calculation only moments of distributions in $x$ can be accessed as matrix elements of local operators $[12,13]$. To overcome these issues, the proposed large-momentum effective theory (LaMET) of Ji has been presented [14]. This method evaluates PDFs on the lattice through quasi-PDFs [14-16], whose mother correlation functions includes a spacelike operator $\gamma^{z}$ instead of the usual lightlike $\gamma^{+}$entering the definition of the standard PDFs. These quasi-PDFs can be reached directly from the lattice QCD calculation [17] and as the quasi-PDFs depend logarithmically on $P_{z}$ when $P_{z}$ becomes large, and they need a perturbative matching in LaMET to reduce to the standard PDFs. A very recent review of LaMET is given in [18]. Many theoretical discussions and lattice simulations for quasi-PDFs 
and similar quantities has been performed [19-34]. Moreover, several model calculations of quasi-PDFs have been carried out [35-41]. There have also been a number of works on quasi-PDFs renormalization [20,42-49]. The approach [14] can be generalized to any light-cone correlations in hadron physics, e.g. the correlators related to GPDs and TMDs. There has been a lot of efforts on the quasi-TMDs, including their renormalization and matching to the physical TMDs [18,50-58]. These quasi-TMD works have made important breakthroughs on their pinched-pole singularity issue, renormalization, evolution, soft factor subtraction, and factorization into physical TMDs. Moreover, the nonperturbative Collins-Soper evolution kernel of TMDs has been calculated in $[59,60]$, which is an important step in TMDs full extractions from lattice QCD.

Among the hadrons, pions are very fascinating particles and they hold a lot of information on the structure of hadrons. There has been a tremendous effort to deduce the parton distribution functions of the pion. Pions provide the force that binds the protons and neutrons inside the nuclei and they also influence the properties of the isolated nucleons. Thus understanding of matter is not complete without getting a detailed information on the role of pions. In this paper, being inspired by the previous works for quasi-distribution model calculations [61-63], we will probe the T-odd GTMDs, quasi-TMDs and the quasi-GPDs of the pion applying a scalar spectator model. In particular, GPDs of the pion have been obtained in various models like chiral quark model [64,65], NJL model [66,67], light-front constituent quark model [68] and lattice QCD [69-72]. We will give out the analytical results of all four twist-two T-odd GTMDs, quasi-TMDs and quasi-GPDs in the present paper, and conduct a qualitative analysis of all these distributions.

The remainder of this paper is as follows: Sect. 2 below describes in detail the theoretical definition of various pion parton distributions. In Sect. 3 we give out the analytical results of four T-odd GTMDs, quasi-TMDs and quasi-GPDs in a scalar spectator. In Sect. 4, we present our numerical studies using a group of fitted model parameters of a scalar spectator model. A brief conclusion is presented in Sect. 5 .

\section{Definition of pion parton distributions}

\subsection{Transverse momentum dependent parton distribution $h_{1 \pi}^{\perp}$}

For a spinless particle, such as the pion, only two leading twist TMDs arise, in contrast to the eight found for spin$\frac{1}{2}$ particles [73]. The TMD $f_{1 \pi}$ is simply the unpolarized quark distribution, whereas the Boer-Mulders (BM) function [74], $h_{1 \pi}^{\perp}$, describes the distribution of transversely polarized quarks in the pion. The BM function $h_{1 \pi}^{\perp}$ is defined from the quark-quark distribution correlation function

$$
\begin{aligned}
\Phi^{[\Gamma]}\left(x, \vec{k}_{T}\right)= & \int \frac{1}{2} \frac{d \xi^{-} d^{2} \vec{\xi}_{T}}{(2 \pi)^{3}} e^{i k \cdot \xi}|P| \bar{\psi}\left(-\frac{1}{2} \xi\right) \Gamma \mathcal{W}_{+\infty} \\
& \times\left.\left(-\frac{1}{2} \xi ; \frac{1}{2} \xi\right) \psi\left(\frac{1}{2} \xi\right)|P\rangle\right|_{\xi^{+}=0}
\end{aligned}
$$

where $P$ is the four-momentum of the pion moving along the $z$-axis with the components $\left(P^{+}, P^{-}, \overrightarrow{0}_{\perp}\right)$ in the light-cone coordinates, in which the plus and minus components of any four-vector $a^{\mu}$ have the form $a^{ \pm}=\left(a^{0} \pm a^{3}\right) / \sqrt{2}$, and transverse part $\vec{a}_{\perp}=\left(a^{1}, a^{2}\right)$. The quark field and momentum are denoted by $k$ and $\psi$. In the correlator Eq. (1) we have Wilson lines

$$
\begin{aligned}
&\left.\mathcal{W}_{+\infty}\left(-\frac{1}{2} \xi ; \frac{1}{2} \xi\right)\right|_{\xi}=0 \\
&= {\left[0^{+},-\frac{1}{2} \xi^{-},-\frac{1}{2} \vec{\xi}_{T} ; 0^{+},+\infty^{-},\right.} \\
&\left.-\frac{1}{2} \vec{\xi}_{T}\right] \cdot\left[0^{+},+\infty^{-},-\frac{1}{2} \vec{\xi}_{T} ; 0^{+},+\infty^{-}, \frac{1}{2} \vec{\xi}_{T}\right] \\
& \cdot\left[0^{+},+\infty^{-}, \frac{1}{2} \vec{\xi}_{T} ; 0^{+}, \frac{1}{2} \xi^{-}, \frac{1}{2} \vec{\xi}_{T}\right],
\end{aligned}
$$

where

$$
\begin{aligned}
& {\left[0^{+},-\frac{1}{2} \xi^{-},-\frac{1}{2} \vec{\xi}_{T} ; 0^{+},+\infty^{-},-\frac{1}{2} \vec{\xi}_{T}\right]} \\
& =\mathcal{P} \exp \left[-i g_{s} \int_{-\frac{1}{2} \xi^{-}}^{+\infty^{-}} d \zeta A^{+}\left(\zeta^{-}, 0^{+},-\frac{1}{2} \vec{\xi}_{T}\right)\right] \\
& {\left[0^{+},+\infty^{-},-\frac{1}{2} \vec{\xi}_{T} ; 0^{+},+\infty^{-}, \frac{1}{2} \vec{\xi}_{T}\right]} \\
& =\mathcal{P} \exp \left[-i g_{s} \int_{-\frac{1}{2} \vec{\xi}_{T}}^{\frac{1}{2} \vec{\xi}_{T}} d \zeta_{T} A_{T}\left(+\infty^{-}, 0^{+}, \vec{\zeta}_{T}\right)\right] \\
& {\left[0^{+},+\infty^{-}, \frac{1}{2} \vec{\xi}_{T} ; 0^{+}, \frac{1}{2} \xi^{-}, \frac{1}{2} \vec{\xi}_{T}\right]} \\
& =\mathcal{P} \exp \left[-i g_{s} \int_{+\infty^{-}}^{\frac{1}{2} \xi^{-}} d \zeta A^{+}\left(\zeta^{-}, 0^{+}, \frac{1}{2} \vec{\xi}_{T}\right)\right] .
\end{aligned}
$$

Here $\mathcal{P}$ is the path-ordering operator and $A$ is the gluon field. The strong couping constant is denoted by $g_{s}$. The BM function $h_{1 \pi}^{\perp}$ can be obtained by

$$
\Phi^{\left[i \sigma^{\alpha+} \gamma_{5}\right]}=-\frac{\varepsilon_{T}^{\alpha \beta} k_{T}^{\beta}}{M} h_{1 \pi}^{\perp}
$$

with the pion mass denoted by $M$.

\subsection{T-odd GTMDs and GPDs}

To obtain GTMDs, we start from the generalized $k_{T}$ dependent correlator denoted by 


$$
\begin{aligned}
W^{[\Gamma]}\left(x, \vec{k}_{T}, \Delta\right)= & \int \frac{1}{2} \frac{d \xi^{-} d^{2} \vec{\xi}_{T}}{(2 \pi)^{3}} e^{i k \cdot \xi}\left\langle p^{\prime}\right| \bar{\psi}\left(-\frac{1}{2} \xi\right) \Gamma \mathcal{W}_{+\infty} \\
& \times\left.\left(-\frac{1}{2} \xi ; \frac{1}{2} \xi\right) \psi\left(\frac{1}{2} \xi\right)|p\rangle\right|_{\xi^{+}=0}
\end{aligned}
$$

where the initial and final state four-momentum are characterized by $p$ and $p^{\prime}$. We use the common kinematical variables

$$
\begin{aligned}
P & =\frac{1}{2}\left(p+p^{\prime}\right), \quad \Delta=p^{\prime}-p, \\
t & =\Delta^{2}=-\frac{1}{1-\xi^{2}}\left(4 \xi^{2} M^{2}+\vec{\Delta}_{T}^{2}\right), \\
P^{\mu} & =\left[P^{+}, \frac{4 M^{2}+\vec{\Delta}_{T}^{2}}{8\left(1-\xi^{2}\right) P^{+}}, \overrightarrow{0}_{T}\right], \\
k^{\mu} & =\left[x P^{+}, \frac{k^{2}+\vec{k}_{T}^{2}}{2 x P^{+}}, \vec{k}_{T}\right], \\
\Delta^{\mu} & =\left[-2 \xi P^{+}, \frac{\xi\left(4 M^{2}+\vec{\Delta}_{T}^{2}\right)}{4\left(1-\xi^{2}\right) P^{+}}, \vec{\Delta}_{T}\right],
\end{aligned}
$$

where we consider the range $0 \leq \xi \leq 1$ of the skewness variable $\xi$. In general, the generalized $k_{T}$-dependent correlator in Eq. (5), unlike GPDs or TMDs, are complex-valued functions. We can reach four complex-valued twist-two GTMDs $F_{1}, \tilde{G}_{1}, H_{1}^{k}, H_{1}^{\Delta}$ through

$$
\begin{aligned}
W^{\left[\gamma^{+}\right]}= & F_{1}^{e}+i F_{1}^{o}, \\
W^{\left[\gamma^{+} \gamma_{5}\right]}= & \frac{i \varepsilon_{T}^{i j} k_{T}^{i} \Delta_{T}^{j}}{M^{2}}\left(\tilde{G}_{1}^{e}+i \tilde{G}_{1}^{o}\right), \\
W^{\left[i \sigma^{j+} \gamma_{5}\right]}= & \frac{i \varepsilon_{T}^{i j} k_{T}^{i}}{M}\left(H_{1}^{k, e}+i H_{1}^{k, o}\right) \\
& +\frac{i \varepsilon_{T}^{i j} \Delta_{T}^{i}}{M}\left(H_{1}^{\Delta, o}+i H_{1}^{\Delta, o}\right),
\end{aligned}
$$

where the superscripts $e, o$ stand for T-even and T-odd part respectively. We have adopted the general definitions $\sigma^{\mu \nu}=$ $i\left[\gamma^{\mu}, \gamma^{\nu}\right] / 2, \varepsilon^{0123}=1$ and $\varepsilon_{T}^{i j}=\varepsilon^{-+i j}$. The T-odd twisttwo GTMDs correspond to the imaginary part of complexvalued twist-two GTMDs.

The twist-two standard GPDs of quarks for a spin-0 hadron come from the integrated quark-quark correlator obtained from the correlator $W$ in Eq. (5) by means of the projection

$$
\begin{gathered}
F^{[\Gamma]}(x, \Delta)=\int d k^{-} d^{2} \vec{k}_{T} W^{[\Gamma]}\left(x, \vec{k}_{T}, \Delta\right) \\
=\int \frac{1}{2} \frac{d \xi^{-}}{2 \pi} e^{i k \cdot \xi}\left\langle p^{\prime}\right| \bar{\psi}\left(-\frac{1}{2} \xi\right) \Gamma \mathcal{W} \\
\quad \times\left(-\frac{1}{2} \xi ; \frac{1}{2} \xi\right) \psi\left(\frac{1}{2} \xi\right)|p|_{\xi^{+}=0} .
\end{gathered}
$$

The GPDs parameterize the Dirac traces $F^{[\Gamma]}$ of the GPDcorrelator in Eq. (8) and there are only two GPDs in twist-two

$$
\begin{aligned}
F^{\left[\gamma^{+}\right]} & =F_{1}(x, \xi, t), \\
F^{\left[i \sigma^{j+} \gamma_{5}\right]} & =\frac{i \varepsilon_{T}^{i j} \Delta_{T}^{i}}{M} H_{1}(x, \xi, t) .
\end{aligned}
$$

\subsection{Quasi-TMD and quasi-GPD}

In the following we turn to the definitions of the quasiTMD and quasi-GPD of the pion meson. Quasi-TMD $h_{1 \pi}^{\perp}\left(x, \vec{k}_{T}^{2} ; P_{z}\right)$ is defined through an equal-time spatial correlation function

$$
\begin{aligned}
\Phi^{[\Gamma]}\left(x, \vec{k}_{T} ; P_{z}\right)= & \int \frac{1}{2} \frac{d \xi^{-} d^{2} \vec{\xi}_{T}}{(2 \pi)^{3}} e^{i k \cdot \xi}\langle P| \bar{\psi}\left(-\frac{1}{2} \xi\right) \Gamma \mathcal{W}_{Q,+\infty} \\
& \times\left.\left(-\frac{1}{2} \xi ; \frac{1}{2} \xi\right) \psi\left(\frac{1}{2} \xi\right)|P\rangle\right|_{\xi^{+}=0}
\end{aligned}
$$

The Wilson lines read

$$
\begin{aligned}
& \left.\mathcal{W}_{Q,+\infty}\left(-\frac{1}{2} \xi ; \frac{1}{2} \xi\right)\right|_{\xi^{+}=0}=\left[0,-\frac{1}{2} \xi^{3},\right. \\
& \left.-\frac{1}{2} \vec{\xi}_{T} ; 0,+\infty^{3},-\frac{1}{2} \vec{\xi}_{T}\right] \\
& \cdot\left[0,+\infty^{3},-\frac{1}{2} \vec{\xi}_{T} ; 0,+\infty^{3}, \frac{1}{2} \vec{\xi}_{T}\right] \\
& \cdot\left[0,+\infty^{3}, \frac{1}{2} \vec{\xi}_{T} ; 0, \frac{1}{2} \xi^{3}, \frac{1}{2} \vec{\xi}_{T}\right]
\end{aligned}
$$

where

$$
\begin{aligned}
& {\left[0,-\frac{1}{2} \xi^{3},-\frac{1}{2} \vec{\xi}_{T} ; 0,+\infty^{3},-\frac{1}{2} \vec{\xi}_{T}\right]} \\
& =\mathcal{P} \exp \left[-i g_{s} \int_{-\frac{1}{2} \xi^{3}}^{+\infty^{3}} d \zeta A^{3}\left(\zeta^{3}, 0,-\frac{1}{2} \vec{\xi}_{T}\right)\right], \\
& {\left[0,+\infty^{3},-\frac{1}{2} \vec{\xi}_{T} ; 0,+\infty^{3}, \frac{1}{2} \vec{\xi}_{T}\right]} \\
& =\mathcal{P} \exp \left[-i g_{s} \int_{-\frac{1}{2} \vec{\xi}_{T}}^{\frac{1}{2} \vec{\xi}_{T}} d \zeta_{T} A_{T}\left(+\infty^{3}, 0, \vec{\zeta}_{T}\right)\right], \\
& {\left[0,+\infty^{3}, \frac{1}{2} \vec{\xi}_{T} ; 0, \frac{1}{2} \xi^{3}, \frac{1}{2} \vec{\xi}_{T}\right]} \\
& =\mathcal{P} \exp \left[-i g_{s} \int_{+\infty^{3}}^{\frac{1}{2} \xi^{3}} d \zeta A^{+}\left(\zeta^{3}, 0, \frac{1}{2} \vec{\xi}_{T}\right)\right] .
\end{aligned}
$$

Quasi-TMD $h_{1 \pi, Q}^{\perp}\left(x, \vec{k}_{T}^{2} ; P_{z}\right)$ can be obtained from two definitions

$$
\begin{aligned}
\Phi^{\left[i \sigma^{\alpha 3} \gamma_{5}\right]} & =-\frac{\varepsilon_{T}^{\alpha \beta} k_{T}^{\beta}}{M} h_{1 \pi, Q(3)}^{\perp}\left(x, \vec{k}_{T}^{2} ; P_{z}\right), \\
\Phi^{\left[i \sigma^{\alpha 0} \gamma_{5}\right]} & =-\frac{\varepsilon_{T}^{\alpha \beta} k_{T}^{\beta}}{M} h_{1 \pi, Q(0)}^{\perp}\left(x, \vec{k}_{T}^{2} ; P_{z}\right) .
\end{aligned}
$$


The original paper on quasi-PDFs suggested to use the matrix $\gamma^{3}$ [14] for the unpolarized quasi-PDF $f_{1, Q}\left(x ; P^{3}\right)$. It was later argued that the matrix $\gamma^{0}$ would lead to a better suppression of higher-twist contributions [75]. Similarly, quasiGPDs are also defined through an equal-time spatial correlation function

$$
\begin{aligned}
& F_{Q}^{[\Gamma]}\left(x, \Delta ; P_{z}\right) \\
& =\int \frac{1}{2} \frac{d \xi^{-}}{2 \pi} e^{i k \cdot \xi}\left\langle p^{\prime}\right| \bar{\psi}\left(-\frac{1}{2} \xi\right) \Gamma \mathcal{W}_{Q} \\
& \quad \times\left.\left(-\frac{1}{2} \xi ; \frac{1}{2} \xi\right) \psi\left(\frac{1}{2} \xi\right)|p\rangle\right|_{\xi^{0}=0, \vec{z}_{\perp}=\overrightarrow{0}_{\perp}},
\end{aligned}
$$

where the Wilson line is given by

$$
\begin{aligned}
& \left.\mathcal{W}_{Q}\left(-\frac{1}{2} \xi ; \frac{1}{2} \xi\right)\right|_{\xi^{0}=0, \vec{z}_{\perp}=\overrightarrow{0}_{\perp}} \\
& =\mathcal{P} \exp \left(-i g_{s} \int_{-\frac{\xi^{3}}{2}}^{\frac{\xi^{3}}{2}} d y^{3} A^{3}\left(0, \overrightarrow{0}_{\perp} ; y^{3}\right)\right) .
\end{aligned}
$$

Then the twist-two quasi-GPDs of the pion can be obtained through two definitions

$$
\begin{aligned}
F^{\left[\gamma^{3}\right]} & =F_{1, Q(3)}\left(x, \xi, t ; P_{z}\right), \quad F^{\left[\gamma^{0}\right]} \\
& =F_{1, Q(0)}\left(x, \xi, t ; P_{z}\right), \\
F^{\left[i \sigma^{j 3} \gamma_{5}\right]} & =\frac{i \varepsilon_{T}^{i j} \Delta_{T}^{i}}{M} H_{1, Q(3)}\left(x, \xi, t ; P_{z}\right), \quad F^{\left[i \sigma^{j 0} \gamma_{5}\right]} \\
& =\frac{i \varepsilon_{T}^{i j} \Delta_{T}^{i}}{M} H_{1, Q(0)}\left(x, \xi, t ; P_{z}\right) .
\end{aligned}
$$

\section{Analytical results in a scalar spectator model}

In this section, being inspired by the previous works for quasidistribution model calculations [61-63], we apply a scalar spectator model to reach the analytic results of the quasiTMD $h_{1 \pi}^{\perp}\left(x, \vec{k}_{T}^{2} ; P_{z}\right)$, quasi-GPD $H_{1}\left(x, \xi, t ; P_{z}\right)$ and T-odd GTMDs. In this model, two types of particles have to be considered: the pion target with mass $M$ and the quark or antiquark with mass $m$. A pion field $\phi$ is coupled to a quark and an antiquark using a pseudo-scalar interaction. Including isospin the interaction part of the Lagrangian reads

$\mathcal{L}_{\text {int }}(x)=-i g_{\pi} \bar{\Psi}(x) \gamma_{5} \vec{\tau} \cdot \vec{\phi}(x) \Psi(x)$,

where $g_{\pi}$ is the coupling constant and $\tau_{i}$ are the Pauli matrices. In the work [63], an exponential form factor have been adopted instead of a simple constant $g_{\pi}$ to eliminate the divergences arising after integration over large $k_{T}$. Furthermore the parameters of the spectator model have been determined by the authors of Ref. [63] through fitting the model result of unpolaried parton distribution $f_{1 \pi}(x)$ to the GRV parametrization [76] for the pion. We follow the same pointlike coupling form in [63] as

$$
\begin{aligned}
g_{\pi} & \equiv g_{\pi}\left(k_{T}\right)=g_{\pi}^{\prime} \exp \left(-\frac{\vec{k}_{T}^{2}}{\bar{x}^{\alpha}(1-\bar{x})^{\beta} \lambda^{2}}\right) \\
& \equiv g_{\pi}^{\prime} \exp \left(-\frac{\vec{k}_{T}^{2}}{\Lambda^{2}(x)}\right),
\end{aligned}
$$

where $\bar{x}=|x|$. Here $g_{\pi}^{\prime}, \alpha, \beta$ and $\lambda$ are the model parameters. By choosing the point-like coupling in Eq. (18), the applicable range of $x$ could be $-1<x<1$. This kinematical region is of great interest for quasi-PDFs and quasi-GPDs.

\subsection{Reuslts for TMD $h_{1 \pi}^{\perp}$, GPD $H_{1}$ and T-odd GTMDs}

We first discuss the result for TMD $h_{1 \pi}^{\perp}$. To get nonzero results for these functions requires considering at least oneloop corrections that include effects from the Wilson line. At the leading order in $g_{s}^{2}$, one finds for the correlator in Eq. (1)

$$
\begin{aligned}
& \Phi^{\left[i \sigma^{i+} \gamma_{5}\right]} \\
&= \frac{1}{2} \int d k^{-} \frac{C_{F} g_{s}^{2} g_{\pi}^{2}}{(2 \pi)^{4}} \frac{i(\not k+m)}{k^{2}-m^{2}+i \varepsilon} \gamma_{5} \frac{i(\not P-\not k-m)}{(P-k)^{2}-m^{2}+i \varepsilon} \\
& \cdot i \int \frac{d^{4} l}{(2 \pi)^{4}} \gamma^{+} \frac{-i}{l^{2}+i \varepsilon} \frac{1}{-l^{+}+i \varepsilon} \frac{i(\not k-l+m)}{(k-l)^{2}-m^{2}+i \varepsilon} \\
& \gamma_{5} \frac{i(k k-l-\not P+m)}{(k-l-P)^{2}-m^{2}+i \varepsilon} i \sigma^{i+} \gamma_{5},
\end{aligned}
$$

where $l^{+}$integral is realized from taking the imaginary part of the eikonal propagator: $1 /\left(-l^{+}+i \varepsilon\right) \rightarrow-2 \pi i \delta\left(l^{+}\right)$. The color factor satisfies $C_{F}=4 / 3$. Then performing the integrals for $k^{-}$and $l^{-}$applying contour integration together with Eq. (4), one obtains

$$
\begin{aligned}
& h_{1 \pi}^{\perp}=\frac{-m M}{2} \frac{C_{F} g_{s}^{2} g_{\pi}^{2}}{(2 \pi)^{4}} \frac{1}{\vec{k}_{T}^{2}\left(\vec{k}_{T}^{2}+m^{2}+x(x-1) M^{2}\right)} \\
& \quad \times \ln \left(\frac{\vec{k}_{T}^{2}+m^{2}+M^{2}(x-1) x}{m^{2}+M^{2}(x-1) x}\right)
\end{aligned}
$$

where we have used

$$
\begin{aligned}
& \int d^{2} \vec{l}_{T} \frac{\vec{k}_{T} \cdot \vec{l}_{T}}{\vec{l}_{T}^{2}\left[\left(k_{T}-l_{T}\right)^{2}+m^{2}+M^{2}(x-1) x\right]} \\
& =-\pi \ln \left(\frac{\vec{k}_{T}^{2}+m^{2}+M^{2}(x-1) x}{m^{2}+M^{2}(x-1) x}\right) .
\end{aligned}
$$

The result in Eq. (20) is the same with previous prediction in Refs. [8,77]. $h_{1 \pi}^{\perp}$ is negative which agrees with previous expectations [78].

The GPD $H_{1}$ can be extracted from the integrated quarkquark correlator in Eq. (8), which reads 
$W^{\left[i \sigma^{j+} \gamma_{5}\right]}=\int \frac{d k^{-} d^{2} k_{T}}{2(2 \pi)^{4}} \frac{g_{\pi}^{+} g_{\pi}^{-} \operatorname{Tr}\left[\gamma_{5} i(\not P-\not k-m) \gamma_{5} i\left(\not k+\frac{1}{2} \not \Delta+m\right) i \sigma^{\alpha+} \gamma_{5} i\left(\not k-\frac{1}{2} \not \Delta+m\right)\right]}{\left[(P-k)^{2}-m^{2}+i \varepsilon\right]\left[\left(k-\frac{1}{2} \Delta\right)^{2}-m^{2}+i \varepsilon\right]\left[\left(k+\frac{1}{2} \Delta\right)^{2}-m^{2}+i \varepsilon\right]}$,

$$
W^{\left[i \sigma^{j+} \gamma_{5}\right]}=\int \frac{d k^{-}}{2(2 \pi)^{4}} \frac{g_{\pi}^{+} g_{\pi}^{-} \operatorname{Tr}\left[\gamma _ { 5 } i ( \not P - \not k - m ) \gamma _ { 5 } i \left(\not k+\frac{1}{2} \not\right.\right.}{\left[(P-k)^{2}-m^{2}+i \varepsilon\right]\left[\left(k-\frac{1}{2} \Delta\right)^{2}-m^{2}+i \varepsilon\right]\left[\left(k+\frac{1}{2} \Delta\right)^{2}-m^{2}+i \varepsilon\right]},
$$

where $g_{\pi}^{ \pm}=g_{\pi}\left(k_{T} \pm \frac{1}{2} \Delta_{T}\right)$. Performing the integrals for $k^{-}$applying contour integration together with Eq. (9), we obtains
Using a similar calculation like GPD $H_{1}$ in the above it is straight to find that $H_{1}^{k}=0$ and $H_{1}^{\pi}\left(x, \xi, \Delta_{T}\right)=$

$H_{1}\left(x, \xi, \Delta_{\perp}\right)= \begin{cases}0 & -1 \leq x \leq-\xi, \\ -\frac{g_{\pi}^{\prime 2}(x+\xi)(1+\xi)\left(1-\xi^{2}\right)}{2(2 \pi)^{3}(1-x)} \int d^{2} \vec{k}_{\perp} \frac{m M}{D_{1} D_{2}^{1}} \exp \left(-\frac{2 \vec{k}_{\perp}^{2}+\frac{1}{2} \vec{\Delta}_{\perp}^{2}}{\Lambda^{2}(x)}\right) & -\xi \leq x \leq \xi, \\ -\frac{g_{\pi}^{\prime 2}\left(1-\xi^{2}\right)(1-x)}{(2 \pi)^{3}} \int d^{2} \vec{k}_{\perp} \frac{m M}{D_{1} D_{2}^{2}} \exp \left(-\frac{2 \vec{k}_{\perp}^{2}+\frac{1}{2} \vec{\Delta}_{\perp}^{2}}{\Lambda^{2}(x)}\right) & x \geq \xi,\end{cases}$

where the $D_{i}$ in the denominator has the form

$$
\begin{aligned}
D_{1}= & (1+\xi)^{2} \vec{k}_{\perp}^{2}+\frac{1}{4}(1-x)^{2} \vec{\Delta}_{\perp}^{2}-(1-x)(1+\xi) \vec{k}_{\perp} \cdot \vec{\Delta}_{\perp} \\
& +(1+\xi)^{2} m^{2}-(1-x)(x+\xi) M^{2}, \\
D_{2}^{1}= & \xi\left(1-\xi^{2}\right) \vec{k}_{\perp}^{2}+\frac{1}{4}\left(1-x^{2}\right) \xi \vec{\Delta}_{\perp}^{2} \\
& +x\left(1-\xi^{2}\right) \vec{k}_{\perp} \cdot \vec{\Delta}_{\perp}+\xi\left(1-\xi^{2}\right) m^{2}-\xi\left(x^{2}-\xi^{2}\right) M^{2}, \\
D_{2}^{2}= & (1-\xi)^{2} \vec{k}_{\perp}^{2}+\frac{1}{4}(1-x)^{2} \vec{\Delta}_{\perp}^{2}+(1-x)(1-\xi) \vec{k}_{\perp} \cdot \vec{\Delta}_{\perp} \\
& +(1-\xi)^{2} m^{2}-(1-x)(x-\xi) M^{2} .
\end{aligned}
$$

One can project the GPD $H_{1}$ from the GTMDs by integrating upon transverse parton momentum [8]

$H_{1}^{\pi}\left(x, \xi, \Delta_{T}\right)=\int d^{2} \vec{k}_{T}\left[\frac{\vec{k}_{T} \cdot \vec{\Delta}_{T}}{\Delta_{T}^{2}} H_{1}^{k}+H_{1}^{\Delta}\right]$ $\int d^{2} \vec{k}_{T} H_{1}^{\Delta}$, which meets the projection relation in Eq. (25).

Then we focus on T-odd GTMDs in the spectator model. Similar to TMD case, one needs to introduce one loop diagrams for the correlator in Eq. (5). The correlator in Eq. (5) reads in the spectator model

$$
\begin{aligned}
W^{[\Gamma]}= & \int d k^{-} \frac{C_{F} g_{s}^{2} g_{\pi}^{+} g_{\pi}^{-}}{(2 \pi)^{4}} \cdot i \int \frac{d^{4} l}{(2 \pi)^{4}} \frac{i\left(k-\frac{1}{2} \not \Delta-l+m\right)}{\left(k-\frac{1}{2} \Delta-l\right)^{2}-m^{2}+i \varepsilon} \\
& \gamma_{5} \frac{i(k-\not p-l+m)}{(k-P-l)^{2}-m^{2}+i \varepsilon} \gamma^{+} \cdot \frac{-i}{l^{2}+i \varepsilon} \frac{1}{-l^{+}+i \varepsilon} \\
& \frac{i(\not P-\not k-m)}{(P-k)^{2}-m^{2}+i \varepsilon} \gamma_{5} \frac{i\left(k+\frac{1}{2} \not \Delta+m\right)}{\left(k+\frac{1}{2} \Delta\right)^{2}-m^{2}+i \varepsilon} \Gamma .
\end{aligned}
$$

Evaluating the $k^{-}, l^{-}$-integral by contour integration, the result for the four T-odd GTMDs can be cast into

$$
H^{o}\left(x, \xi, \vec{k}_{T}, \vec{\Delta}_{T}\right)= \begin{cases}0 & -1 \leq x \leq-\xi, \\ 0 & -\xi \leq x \leq \xi, \\ \frac{8 C_{F} g_{s_{\pi}^{2}}^{2}\left(1-\xi^{2}\right)}{(2 \pi)^{5}} \cdot \int d^{2} \vec{l}_{T} \frac{N_{H}}{\vec{l}_{T}^{2} D_{1} D_{2}} \exp \left(-\frac{2 \vec{k}_{\perp}^{2}+\frac{1}{2} \vec{\Delta}_{\perp}^{2}}{\Lambda^{2}(x)}\right) & x \geq \xi,\end{cases}
$$

To extract the GTMDs $H_{1}^{k}$ and $H_{1}^{\Delta}$ through Eq. (7), we should write the corresponding unintegrated quark-quark correlator as where all the four T-odd GTMD only have nonvanishing analytical results in the DGLAP region. The following is a compilation of the numerators of all the leading-twist T-odd GTMDs: 
The denominators in Eq. (28) are given by

$$
\begin{aligned}
D_{1}= & -4 \vec{k}_{T}^{2}(\xi-1)^{2}-4(\xi-1)(x-1) \vec{k}_{T} \\
& \cdot \vec{\Delta}_{T}-(x-1)^{2} \vec{\Delta}_{T}^{2}-4 m^{2}(\xi-1)^{2}-4(x-1)(x-\xi) M^{2}, \\
D_{2}= & -4(\xi+1)^{2} \vec{k}_{T}^{2}+8(\xi+1)^{2} \vec{k}_{T} \cdot \vec{l}_{T}-4(\xi+1)(x-1) \vec{\Delta}_{T}
\end{aligned}
$$

$$
\begin{aligned}
N_{F_{1}^{o}}= & 4\left(\xi^{2}-1\right) \vec{k}_{T}^{2}+4\left(1-\xi^{2}\right) \vec{k}_{T} \cdot \vec{l}_{T}+4 \xi(x-1) \vec{\Delta}_{T} \\
& \vec{k}_{T}-2(\xi+1)(x-1) \vec{\Delta}_{T} \cdot \vec{l}_{T}+4 m^{2}\left(\xi^{2}-1\right) \\
& +(x-1)^{2} \vec{\Delta}_{T}^{2}, \\
N_{\tilde{G}_{1}^{o}}= & -\frac{2 M^{2}}{\vec{\Delta}_{T}^{2} \vec{k}_{T}^{2}}\left(\vec { \Delta } _ { T } ^ { 2 } ( x - 1 ) \left((\xi+1) \vec{l}_{T}\right.\right. \\
& \left.\left.\vec{k}_{T}-2 \vec{k}_{T}^{2}\right)-2 \vec{k}_{T}^{2}\left(\xi^{2}-1\right) \vec{l}_{T} \cdot \vec{\Delta}_{T}\right), \\
N_{H_{1}^{k, o}}= & \frac{4\left(1-\xi^{2}\right) m M \vec{l}_{T} \cdot \vec{k}_{T}}{\vec{k}_{T}^{2}} \\
N_{H_{1}^{\Delta, o}}= & -\frac{4 m M}{\vec{\Delta}_{T}^{2}}\left[\left(\xi^{2}-1\right) \vec{l}_{T} \cdot \vec{\Delta}_{T}+\vec{\Delta}_{T}^{2}(x-1)\right] .
\end{aligned}
$$

where

$$
\begin{aligned}
M_{F_{1}^{o}} & =\frac{(1-\xi) \pi}{1+\xi} \ln \left(\frac{\vec{k}_{T}^{2}+A}{A}\right), \\
M_{\tilde{G}_{1}^{o}} & =\frac{\pi M^{2}(1-x)}{2(\xi+1) \vec{k}_{T}^{2}} \ln \left(\frac{\vec{k}_{T}^{2}+A}{A}\right), \\
M_{H_{1}^{k, o}} & =\frac{\pi m M(1-\xi)}{(1+\xi) \vec{k}_{T}^{2}} \ln \left(\frac{\vec{k}_{T}^{2}+A}{A}\right), \\
M_{H_{1}^{\Delta, o}} & =0
\end{aligned}
$$

with

$$
A=\frac{m^{2}(\xi+1)^{2}+M^{2}(x-1)(\xi+x)}{(\xi+1)^{2}} .
$$

On the other hand, when $\left|\vec{\Delta}_{T}\right| \neq 0$, using the similar method as the derivation of Eq. (32), the the four T-odd GTMD results can be obtained as

$$
H^{o}\left(x, \xi, \vec{k}_{T}, \vec{\Delta}_{T}\right)= \begin{cases}0 & -1 \leq x \leq-\xi, \\ 0 & -\xi \leq x \leq \xi, \\ \frac{8 C_{F} g_{s}^{2} g_{\pi}^{\prime 2}\left(1-\xi^{2}\right)}{(2 \pi)^{5} D_{1}} \exp \left(-\frac{2 \vec{k}_{\perp}^{2}+\frac{1}{2} \vec{\Delta}_{T}^{2}}{\Lambda^{2}(x)}\right) M_{H} B & x \geq \xi,\end{cases}
$$

where

$$
\begin{aligned}
M_{F_{1}^{o}} & =\pi\left(\frac{4\left|\vec{k}_{T}\right|}{2\left|\vec{k}_{T}\right|(\xi+1)+\left|\vec{\Delta}_{T}\right|(x-1)}-1\right), \\
M_{\tilde{G}_{1}^{o}} & =\frac{\pi M^{2}\left(\left|\vec{\Delta}_{T}\right|(1-x)+2\left|\vec{k}_{T}\right|(\xi-1)\right)}{2\left|\vec{\Delta}_{T}\right| \vec{k}_{T}^{2}(\xi+1)+\left|\vec{k}_{T}\right| \vec{\Delta}_{T}^{2}(x-1)}, \\
M_{H_{1}^{k, o}} & =\frac{2 \pi\left|\vec{k}_{T}\right| m M(1-\xi)}{\vec{k}_{T}^{2}\left(2\left|\vec{k}_{T}\right|(1+\xi)+\left|\vec{\Delta}_{T}\right|(x-1)\right)} \\
M_{H_{1}^{\Delta, o}} & =\frac{2 \pi\left|\vec{\Delta}_{T}\right| m M(1-\xi)}{\vec{\Delta}_{T}^{2}\left(2\left|\vec{k}_{T}\right|(1+\xi)+\left|\vec{\Delta}_{T}\right|(x-1)\right)}
\end{aligned}
$$

with

If taking $\left|\vec{\Delta}_{T}\right|=0$, we work out the $\vec{l}_{T}$-integral applying contour theorem and obtain

$$
B=\ln \left(\frac{4 \vec{k}_{T}^{2}(\xi+1)^{2}+4 \vec{\Delta}_{T} \cdot \vec{k}_{T}(\xi+1)(x-1)+4 m^{2}(\xi+1)^{2}+(x-1)\left(4 M^{2}(\xi+x)+2 \vec{\Delta}_{T}^{2}(x-1)\right)}{4 m^{2}(\xi+1)^{2}+(x-1)\left(4 M^{2}(\xi+x)+\vec{\Delta}_{T}^{2}(x-1)\right)}\right) .
$$

$$
H^{o}\left(x, \xi, \vec{k}_{T}, \vec{\Delta}_{T}\right)= \begin{cases}0 & -1 \leq x \leq-\xi, \\ 0 & -\xi \leq x \leq \xi, \\ \frac{8 C_{F} g_{s}^{2} g_{\pi}^{\prime 2}\left(1-\xi^{2}\right)}{(2 \pi)^{5} D_{1}} \exp \left(-\frac{2 \vec{k}_{\perp}^{2}}{\Lambda^{2}(x)}\right) M_{H} & x \geq \xi,\end{cases}
$$



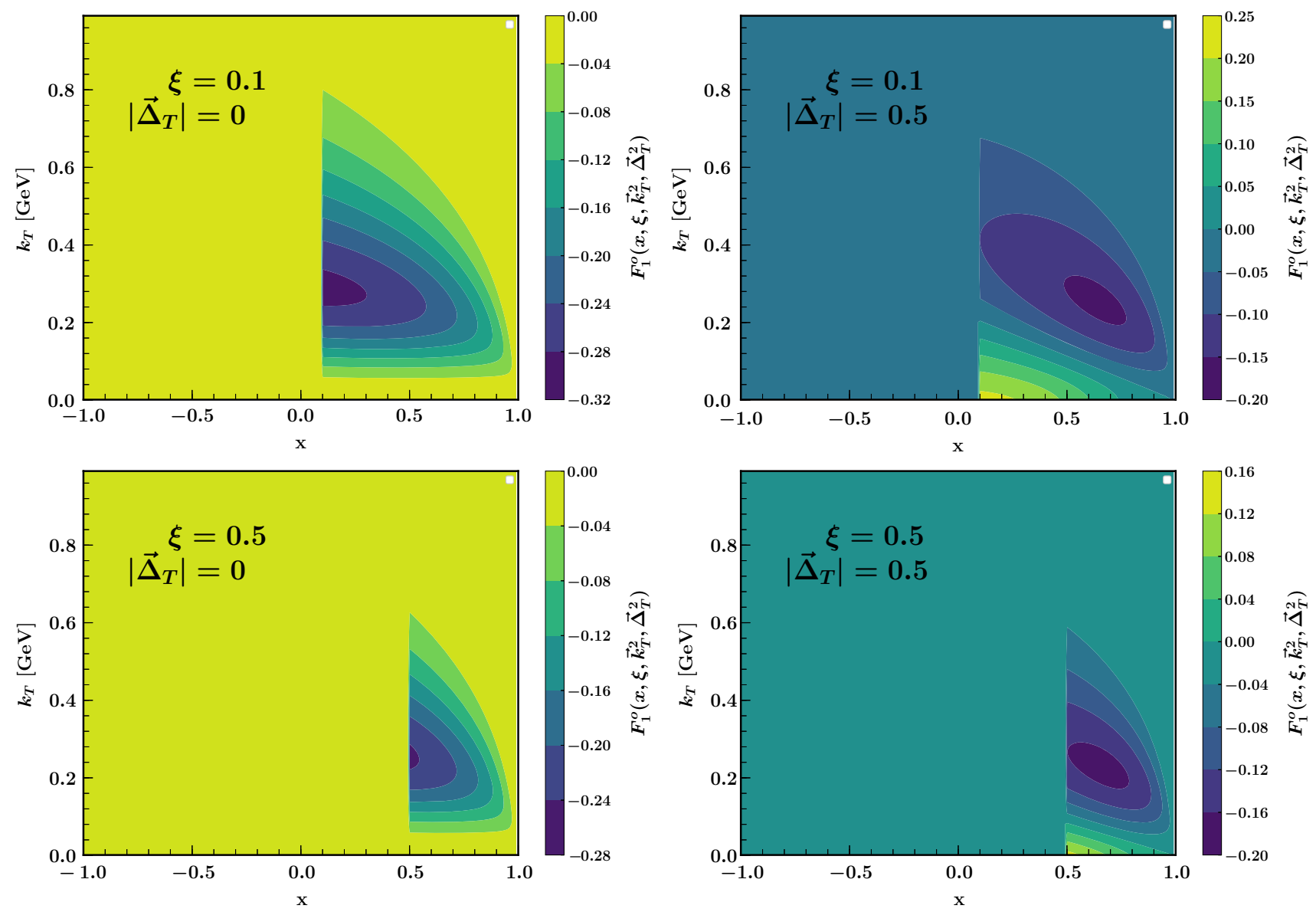

Fig. 1 The T-odd GTMD $F_{1}^{o}\left(x, \xi, \vec{k}_{T}^{2}, \vec{\Delta}_{T}^{2}\right)$ as functions of $x$ and $\left|\vec{k}_{T}\right|$ for different $\xi$ and $\left|\vec{\Delta}_{T}\right|$ values. The upper and lower panels display $F_{1}^{o}\left(x, \xi, \vec{k}_{T}^{2}, \vec{\Delta}_{T}^{2}\right)$ at $\xi=0.1$ and 0.5 , respectively. The left and right panels $F_{1}^{o}\left(x, \xi, \vec{k}_{T}^{2}, \vec{\Delta}_{T}^{2}\right)$ at $\left|\vec{\Delta}_{T}\right|=0$ and 0.5 , respectively

\subsection{Results for quasi-TMD and quasi-GPD}

We start from the equal-time spatial correlation function in Eq. (10), which can be written in the spectator as

$$
\begin{aligned}
& \Phi^{\left[i \sigma^{i 3} \gamma_{5}\right]}=\frac{1}{2} \int d k^{0} \frac{C_{F} g_{s}^{2}}{(2 \pi)^{4}} \frac{i(\not k+m)}{k^{2}-m^{2}+i \varepsilon} \\
& \gamma_{5} \frac{i(\not P-\not k-m)}{(P-k)^{2}-m^{2}+i \varepsilon} \\
& . i \int \frac{d^{4} l}{(2 \pi)^{4}} \gamma^{+} \frac{-i}{l^{2}+i \varepsilon} \frac{1}{-l^{+}+i \varepsilon} \frac{i(\not k-l+m)}{(k-l)^{2}-m^{2}+i \varepsilon} \\
& \gamma_{5} \frac{i(k k-l-\not P+m)}{(k-l-P)^{2}-m^{2}+i \varepsilon} i \sigma^{i 3} \gamma_{5} .
\end{aligned}
$$

In order to apply contour integration we can rewrite the denominator as

$$
\begin{aligned}
& \frac{1}{\left(k^{2}-m^{2}+i \varepsilon\right)\left((P-k)^{2}-m^{2}+i \varepsilon\right)} \\
& \equiv \frac{1}{\left(k^{0}-k_{-}^{0}\right)\left(k^{0}-k_{+}^{0}\right)\left(k^{0}-k_{-}^{0}\right)\left(k^{0}-k_{+}^{0}\right)},
\end{aligned}
$$

where $k_{ \pm}^{0}, k_{ \pm^{\prime}}^{0}$ are the poles for $k^{0}$

$$
\begin{aligned}
& k_{ \pm}^{0}= \pm \sqrt{x^{2} P_{z}^{2}+\vec{k}_{T}^{2}+m^{2}-i \varepsilon}, \\
& k_{ \pm^{\prime}}^{0}=P_{0} \pm \sqrt{(1-x)^{2} P_{z}^{2}+\vec{k}_{T}^{2}+m^{2}-i \varepsilon} .
\end{aligned}
$$

Then according to Eq. (13), the quasi-TMD reads

$$
\begin{aligned}
& h_{1 \pi, Q(3)}^{\perp}\left(x, \vec{k}_{T}^{2} ; P_{z}\right)=-\frac{2 \sqrt{2} m M C_{F} g_{\pi}^{2} \alpha_{s}}{(2 \pi)^{4} \vec{k}_{T}^{2}} . \\
& \quad \times \ln \left(\frac{\vec{k}_{T}^{2}+m^{2}+M^{2}(x-1) x}{m^{2}+M^{2}(x-1) x}\right) \\
& \quad \cdot\left[\frac{1}{\left(k_{-}^{0}-k_{+}^{0}\right)\left(k_{-}^{0}-k_{-^{\prime}}^{0}\right)\left(k_{-}^{0}-k_{+}^{0}\right)}\right. \\
& \left.\quad+\frac{1}{\left(k_{-}^{0}-k_{-}^{0}\right)\left(k_{-^{\prime}}^{0}-k_{+}^{0}\right)\left(k_{-}^{0}-k_{+^{\prime}}^{0}\right)}\right] P^{+},
\end{aligned}
$$

where $P^{+}=1 / \sqrt{2}\left(\sqrt{P_{z}^{2}+M^{2}}+P_{z}\right)$ and $P_{0}=\sqrt{P_{z}^{2}+M^{2}}$. It can be verified that in the limit $P_{z} \rightarrow \infty$, the quasi-TMD in Eq. (41) reduces to the standard TMD shown as Eq. (20). At the same time, $h_{1 \pi, Q(0)}^{\perp}\left(x, \vec{k}_{T}^{2} ; P_{z}\right)=-h_{1 \pi, Q(3)}^{\perp}\left(x, \vec{k}_{T}^{2} ; P_{z}\right)$. 

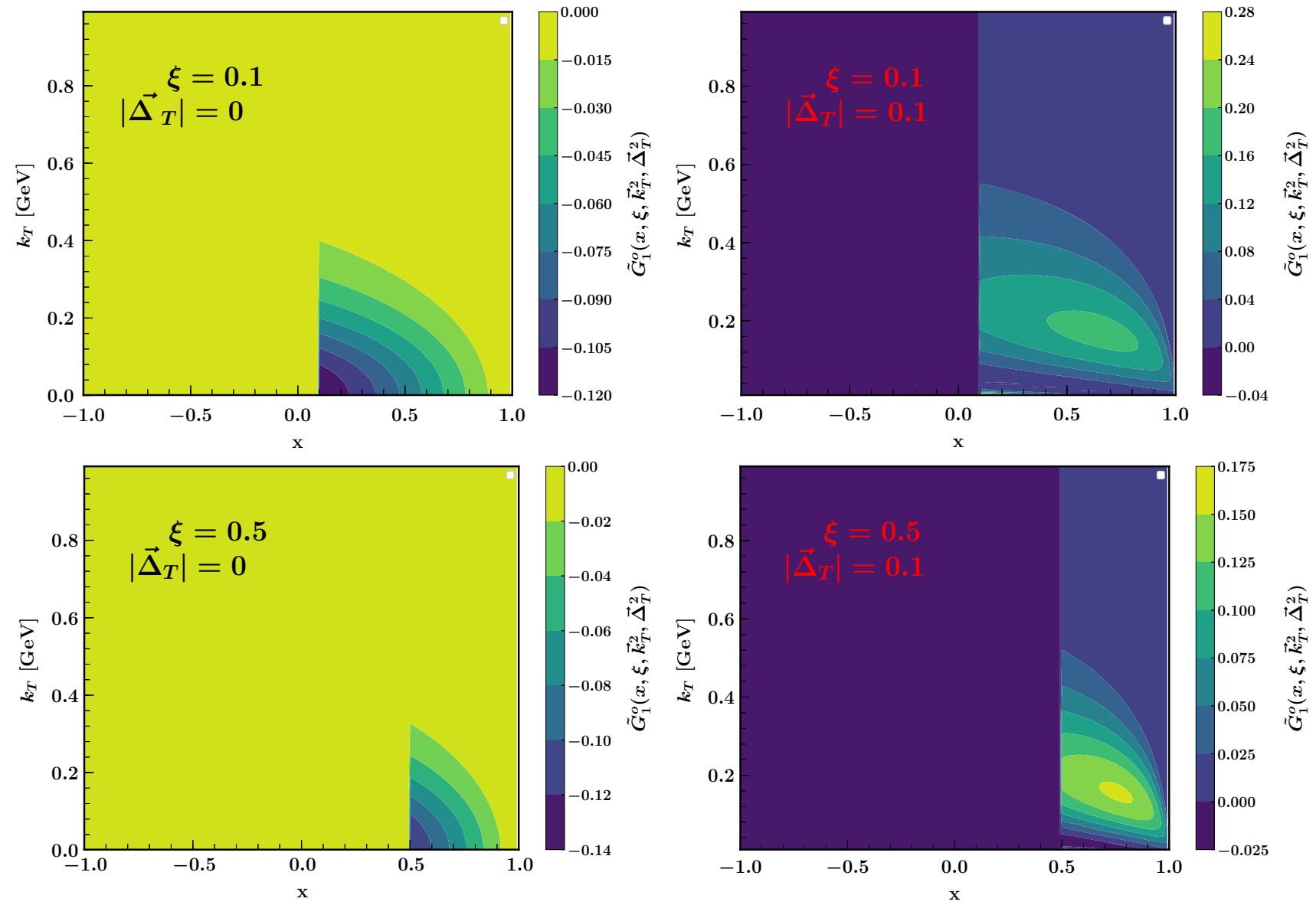

Fig. 2 The T-odd GTMD $\tilde{G}_{1}^{o}\left(x, \xi, \vec{k}_{T}^{2}, \vec{\Delta}_{T}^{2}\right)$ as functions of $x$ and $\left|\vec{k}_{T}\right|$ for different $\xi$ and $\left|\vec{\Delta}_{T}\right|$ values. The upper and lower panels display $\tilde{G}_{1}^{o}\left(x, \xi, \vec{k}_{T}^{2}, \vec{\Delta}_{T}^{2}\right)$ at $\xi=0.1$ and 0.5 , respectively. The left and right panels $\tilde{G}_{1}^{o}\left(x, \xi, \vec{k}_{T}^{2}, \vec{\Delta}_{T}^{2}\right)$ at $\left|\vec{\Delta}_{T}\right|=0$ and 0.1 , respectively

The quasi-GPD of the pion meson can be calculated in a similar way. In the spectator model, the correlator in Eq. (14) to calculate the quasi-GPD has the form:

$$
\begin{aligned}
& \left.-k_{3+}^{0}\right)\left(k_{1-}^{0}-k_{3-}^{0}\right), \\
& D_{2}=\left(k_{2-}^{0}-k_{1+}^{0}\right)\left(k_{2-}^{0}-k_{1-}^{0}\right)\left(k_{2-}^{0}-k_{2+}^{0}\right)\left(k_{2-}^{0}\right. \\
& \left.-k_{3+}^{0}\right)\left(k_{2-}^{0}-k_{3-}^{0}\right),
\end{aligned}
$$$$
\begin{aligned}
W^{\left[i \sigma^{\alpha 3} \gamma_{5}\right]} & =-\int \frac{d k_{0} d^{2} \vec{k}_{T}}{2(2 \pi)^{4}} \frac{g_{\pi}^{+} g_{\pi}^{-} \operatorname{Tr}\left[(\not P-\not k+m)\left(\not k+\frac{1}{2} \Delta+m\right) \sigma^{\alpha 3} \gamma_{5}\left(\not k-\frac{1}{2} \Delta+m\right)\right]}{\left[(P-k)^{2}-m^{2}+i \varepsilon\right]\left[\left(k-\frac{1}{2} \Delta\right)^{2}-m^{2}+i \varepsilon\right]\left[\left(k+\frac{1}{2} \Delta\right)^{2}-m^{2}+i \varepsilon\right]} \\
& =-\int \frac{d k_{0} d^{2} \vec{k}_{T}}{2(2 \pi)^{4}} \frac{g_{\pi}^{+} g_{\pi}^{-} \operatorname{Tr}\left[(\not P-\not k+m)\left(\not k+\frac{1}{2} \Delta+m\right) \sigma^{\alpha 3} \gamma_{5}\left(\not k-\frac{1}{2} \Delta+m\right)\right]}{\left(k^{0}-k_{1-}^{0}\right)\left(k^{0}-k_{1+}^{0}\right)\left(k^{0}-k_{2-}^{0}\right)\left(k^{0}-k_{2+}^{0}\right)\left(k^{0}-k_{3-}^{0}\right)\left(k^{0}-k_{3+}^{0}\right)} . \quad(42)
\end{aligned}
$$

After performing the $k^{0}$-integral using the contour integration, we write down the analytical result of the quasi-GPD as follows according to Eq. (16)

$H_{1, Q(3)}\left(x, \xi, \Delta_{T} ; P_{z}\right)=\int d^{2} \vec{k}_{T} \frac{4 g_{\pi}^{+} g_{\pi}^{-} m M \delta P_{z}}{2(2 \pi)^{3}} \sum_{i=0}^{3} \frac{1}{D_{i}}$,

where

$$
D_{1}=\left(k_{1-}^{0}-k_{1+}^{0}\right)\left(k_{1-}^{0}-k_{2+}^{0}\right)\left(k_{1-}^{0}-k_{2-}^{0}\right)\left(k_{1-}^{0}\right.
$$

$$
\begin{gathered}
D_{3}=\left(k_{3-}^{0}-k_{1+}^{0}\right)\left(k_{3-}^{0}-k_{1-}^{0}\right)\left(k_{3-}^{0}\right. \\
\left.-k_{2+}^{0}\right)\left(k_{3-}^{0}-k_{2-}^{0}\right)\left(k_{3-}^{0}-k_{3+}^{0}\right) .
\end{gathered}
$$

The poles coming from the denominator are given by

$$
k_{1 \pm}^{0}=\delta P_{z} \pm \sqrt{(1-x)^{2} P_{z}^{2}+\vec{k}_{T}^{2}+m^{2}-i \varepsilon}
$$

$$
k_{2 \pm}^{0}=-\xi P_{z} \pm \sqrt{(x+\delta \xi)^{2} P_{z}^{2}+\left(k_{T}-\frac{\Delta_{T}}{2}\right)^{2}+m^{2}-i \varepsilon},
$$



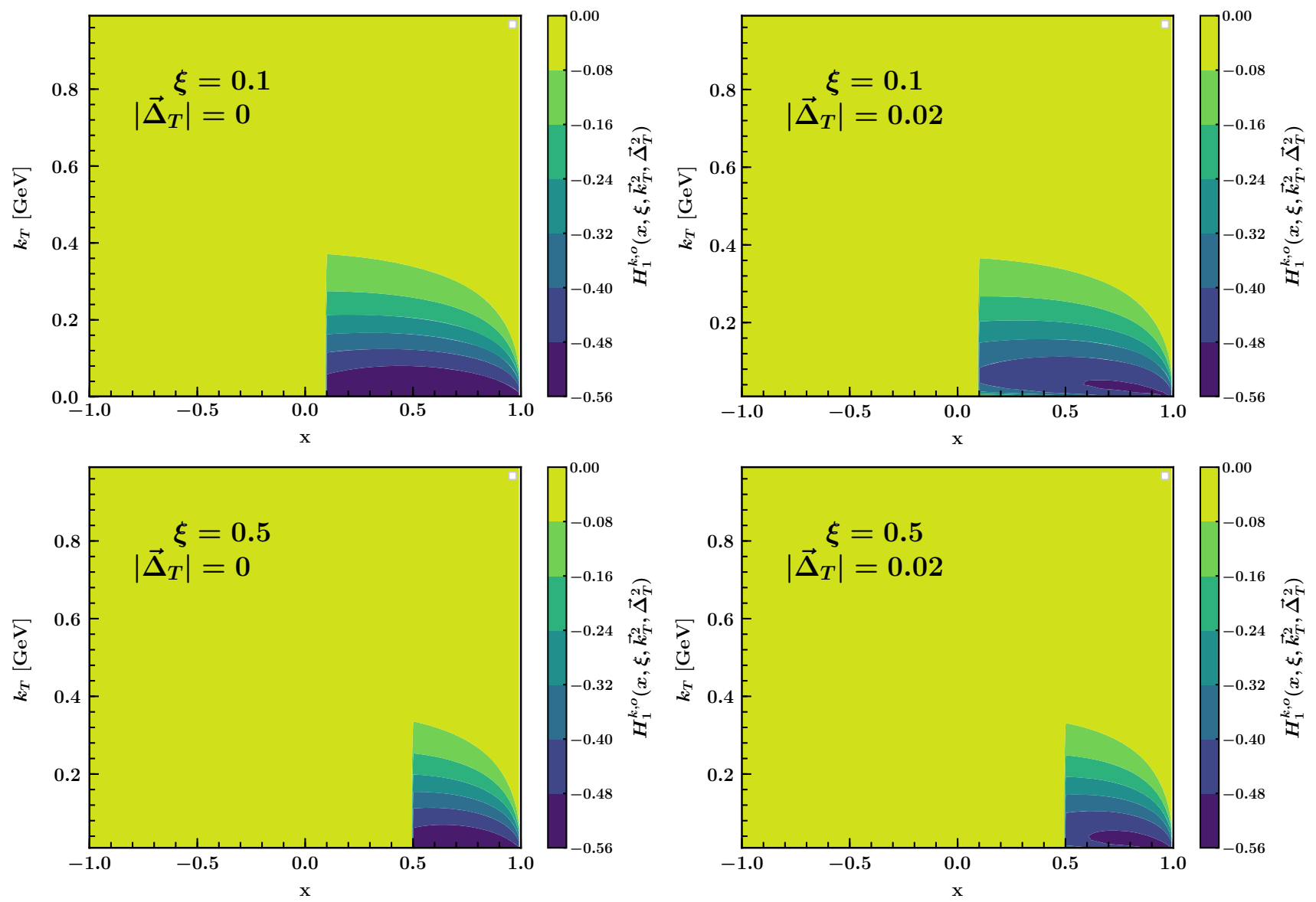

Fig. 3 The T-odd GTMD $H_{1}^{k, o}\left(x, \xi, \vec{k}_{T}^{2}, \vec{\Delta}_{T}^{2}\right)$ as functions of $x$ and $\left|\vec{k}_{T}\right|$ for different $\xi$ and $\left|\vec{\Delta}_{T}\right|$ values. The upper and lower panels display $H_{1}^{k, o}\left(x, \xi, \vec{k}_{T}^{2}, \vec{\Delta}_{T}^{2}\right)$ at $\xi=0.1$ and 0.5 , respectively. The left and right panels $H_{1}^{k, o}\left(x, \xi, \vec{k}_{T}^{2}, \vec{\Delta}_{T}^{2}\right)$ at $\left|\vec{\Delta}_{T}\right|=0$ and 0.02 , respectively
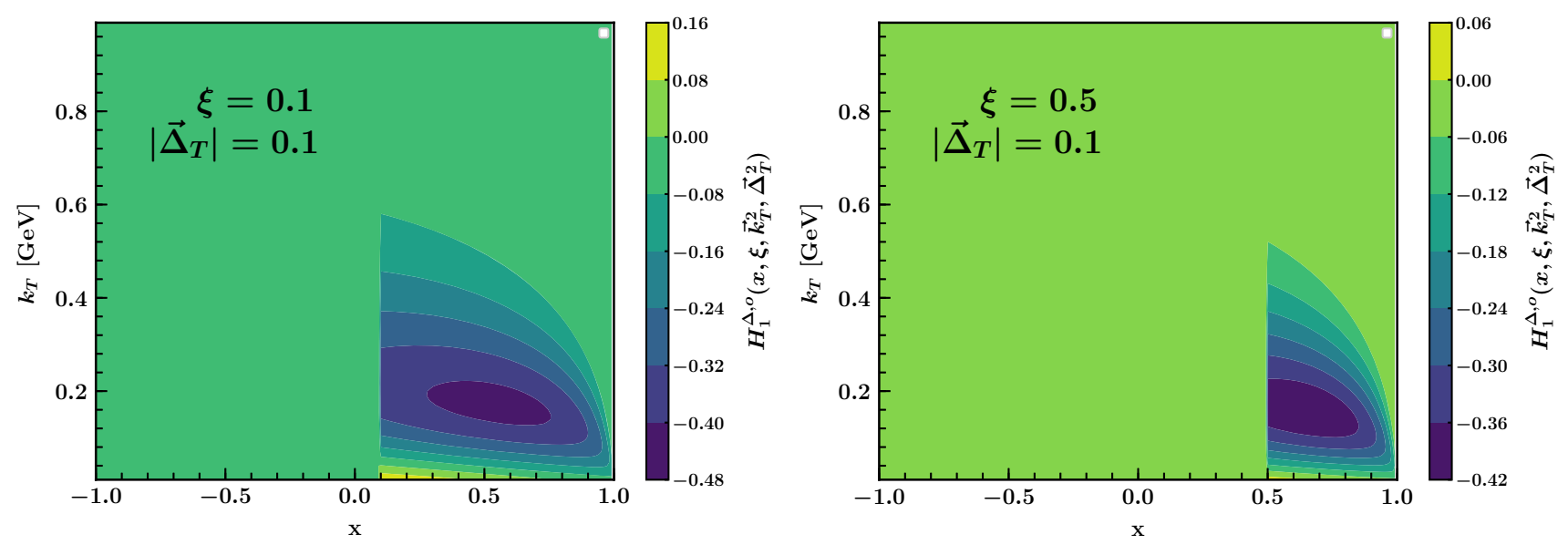

Fig. 4 The T-odd GTMD $H_{1}^{\Delta, o}\left(x, \xi, \vec{k}_{T}^{2}, \vec{\Delta}_{T}^{2}\right)$ as functions of $x$ and $\left|\vec{k}_{T}\right|$ for different $\xi$ values. The left and right panels $H_{1}^{\Delta, o}\left(x, \xi, \vec{k}_{T}^{2}, \vec{\Delta}_{T}^{2}\right)$ at $\left|\vec{\Delta}_{T}\right|=0.1$ with $\xi=0.1$ and 0.5 , respectively 

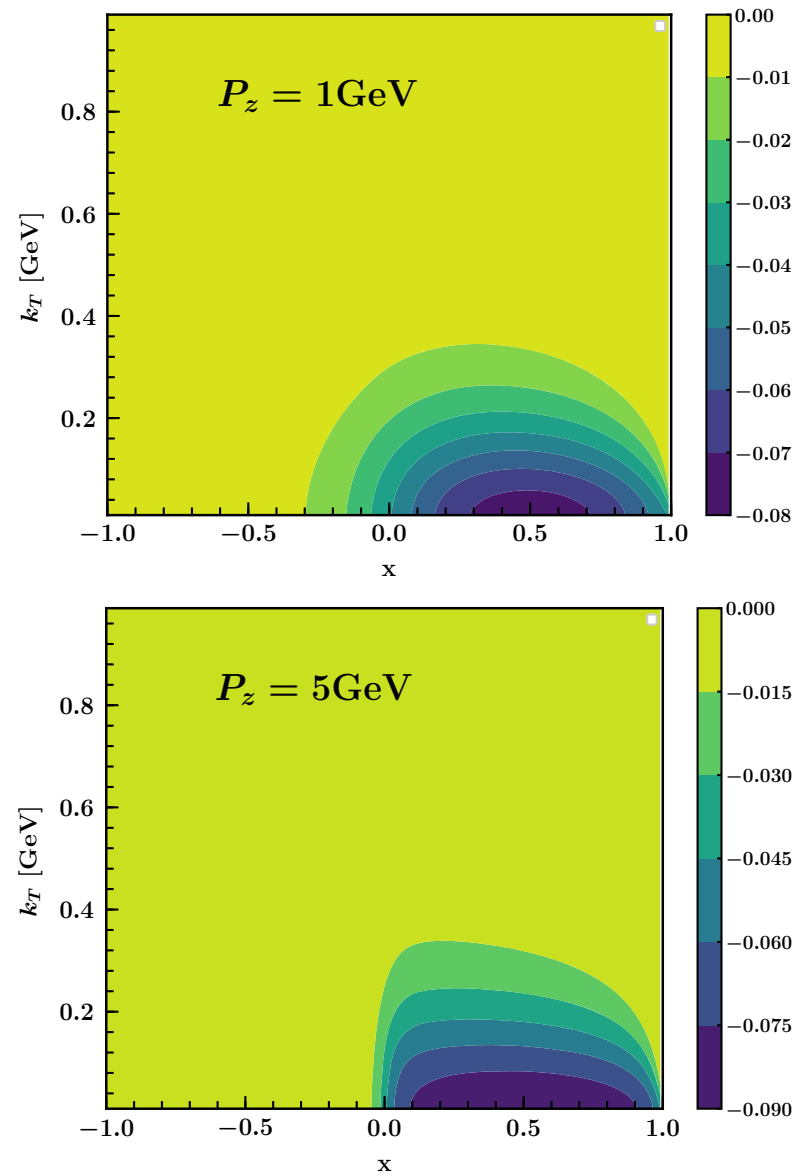

Fig. 5 Quasi-TMD $h_{1 \pi, Q(3)}^{\perp}\left(x, \vec{k}_{T}^{2} ; P_{z}\right)$ as functions of $\mathrm{x}$ and $\left|\vec{k}_{T}\right|$ at different values of $P_{z}$ and TMD $h_{1 \pi}^{\perp}\left(x, \vec{k}_{T}^{2}\right)$ as functions of $\mathrm{x}$ and $\left|\vec{k}_{T}\right|$. Upper left panel: $h_{1 \pi, Q(3)}^{\perp}\left(x, \vec{k}_{T}^{2} ; P_{z}\right)$ at $P_{z}=1 \mathrm{GeV}$. Upper

$$
k_{3 \pm}^{0}=\xi P_{z} \pm \sqrt{(x-\delta \xi)^{2} P_{z}^{2}+\left(k_{T}+\frac{\Delta_{T}}{2}\right)^{2}+m^{2}-i \varepsilon},
$$

with $\delta=P_{0} / P_{z}=1 / P_{z} \sqrt{-t / 4+P_{z}^{2}+M^{2}}$. Similarly, $H_{1, Q(0)}\left(x, \xi, \Delta_{T} ; P_{z}\right)=\frac{1}{\delta} H_{1, Q(3)}\left(x, \xi, \Delta_{T} ; P_{z}\right)$. When $P_{z} \rightarrow \infty$, we have verified that the analytical result in Eq. (43) of the quasi-GPD can be reduced to the light-cone GPD result in Eq. (23).

\section{Numerical calculation}

In order to fix the parameters of the spectator model, the authors of Ref. [63] fit the model result of unpolaried parton distribution $f_{1 \pi}(x)$ to the GRV parametrization [76] for the pion. We adopt the fitted values for the parameters $g_{\pi}^{\prime}=6.316, \lambda=0.855, \alpha=0$ and $\beta=1$. We make a pre-
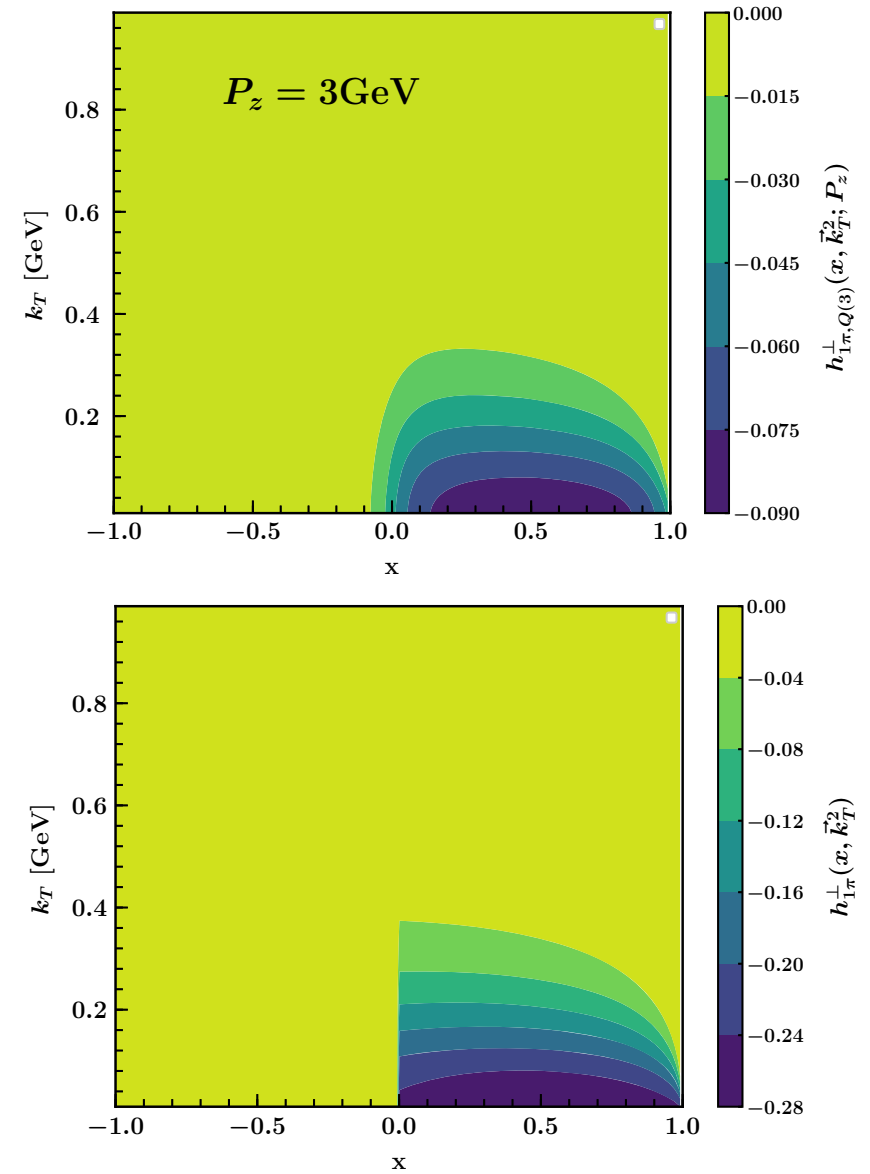

right panel: $h_{1 \pi, Q(3)}^{\perp}\left(x, \vec{k}_{T}^{2} ; P_{z}\right)$ at $P_{z}=3 \mathrm{GeV}$. Lower left panel: $h_{1 \pi, Q(3)}^{\perp}\left(x, \vec{k}_{T}^{2} ; P_{z}\right)$ at $P_{z}=5 \mathrm{GeV}$. Lower right panel: $\operatorname{TMD} h_{1 \pi}^{\perp}\left(x, \vec{k}_{T}^{2}\right)$ as functions of $\mathrm{x}$ and $\left|\vec{k}_{T}\right|$

liminary estimate for choosing the strong coupling $\alpha_{s} \approx 0.3$ and adopting the quark mass $m=0.3 \mathrm{GeV}$.

Firstly, we depict the four T-odd GTMD results considering different values of $\xi$ and $\vec{\Delta}_{T}$ in Figs. 1, 2, 3 and 4 . The T-odd GTMD $F_{1}^{o}\left(x, \xi, \vec{k}_{T}^{2}, \vec{\Delta}_{T}^{2}\right)$ as functions of $x$ and $\vec{k}_{T}$ are shown in Fig. 1. According to Eq. (28), only in $x>\xi$ region, the T-odd GTMD has nonvanishing value. In the upper, left panel of Fig. 1, we plot the T-odd GTMD $F_{1}^{o}\left(x, \xi, \vec{k}_{T}^{2}, \vec{\Delta}_{T}^{2}\right)$ at $\xi=0.1$ and $\left|\vec{\Delta}_{T}\right|=0$. Note that in this case the result of $F_{1}^{o}\left(x, \xi, \vec{k}_{T}^{2}, \vec{\Delta}_{T}^{2}\right)$ is negative. One can also find that as the $x$ value becomes larger, the maximum value of $k_{T}$ resulting in nonvanishing $F_{1}^{o}\left(x, \xi, \vec{k}_{T}^{2}, \vec{\Delta}_{T}^{2}\right)$ becomes smaller and at $x=0.1$, the maximum value of $k_{T}$ is about $0.8 \mathrm{GeV}$. For a fixed $x$ value, as the value of $k_{T}$ increases, the $F_{1}^{o}\left(x, \xi, \vec{k}_{T}^{2}, \vec{\Delta}_{T}^{2}\right)$ result becomes smaller first and then larger. At a fixed value $x=0.1$, the $F_{1}^{o}\left(x, \xi, \vec{k}_{T}^{2}, \vec{\Delta}_{T}^{2}\right)$ reachs the minimum when $k_{T}$ is about 0.3 . We depict the $F_{1}^{o}\left(x, \xi, \vec{k}_{T}^{2}, \vec{\Delta}_{T}^{2}\right)$ at $\xi=0.1$ and $\left|\vec{\Delta}_{T}\right|=0.5$ in the upper, right panel of Fig. 1. Roughly speaking, it can be seen that 

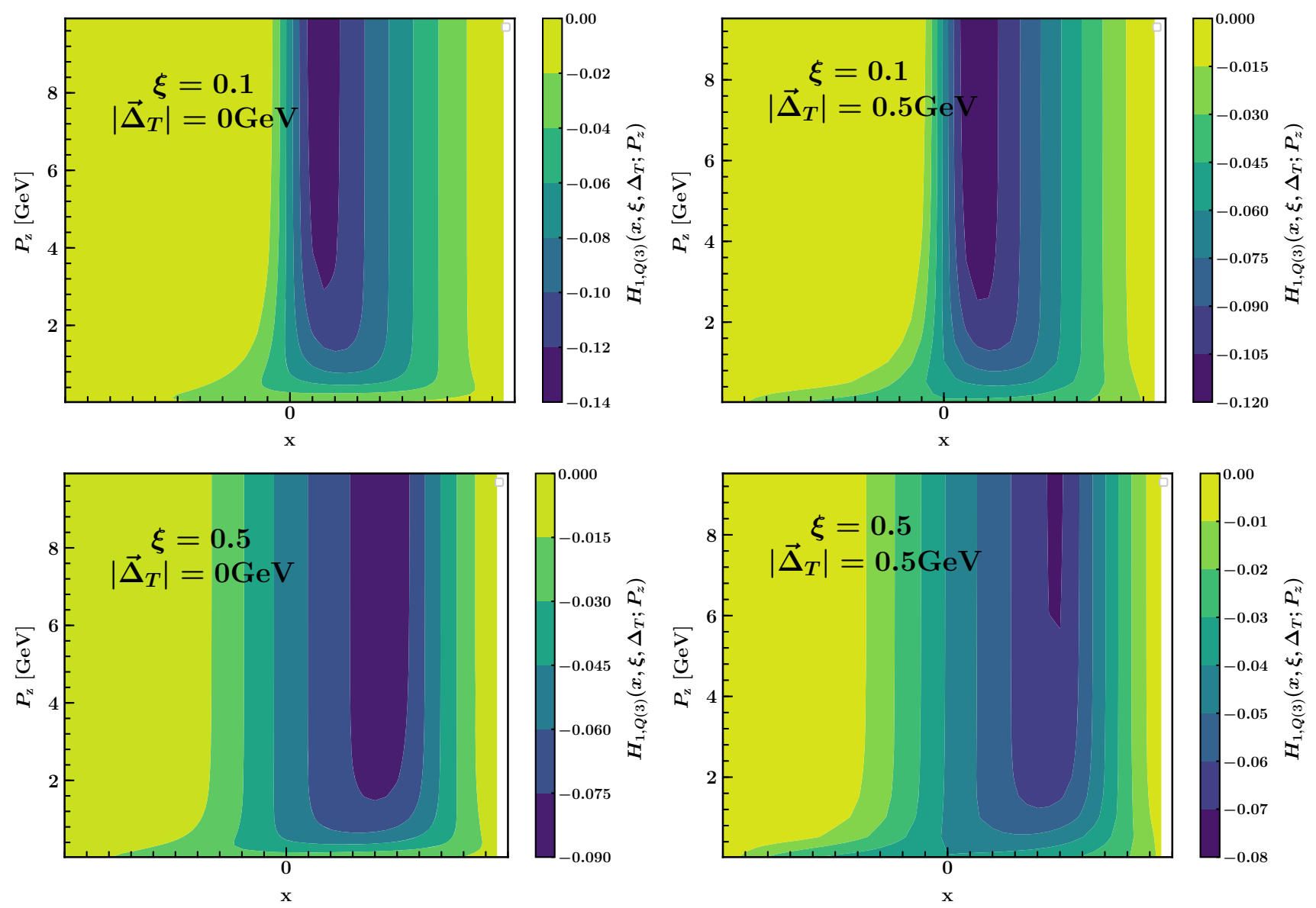

Fig. 6 Quasi-GPD $H_{1, Q(3)}\left(x, \xi, \vec{\Delta}_{T}^{2} ; P_{z}\right)$ as functions of $x$ and $P_{z}$ for different $\xi$ and $\left|\vec{\Delta}_{T}\right|$ values. The upper and lower panels display $H_{1, Q(3)}\left(x, \xi, \vec{\Delta}_{T}^{2} ; P_{z}\right)$ at $\xi=0.1$ and 0.5 , respectively. The left and right panels show $H_{1, Q(3)}\left(x, \xi, \vec{\Delta}_{T}^{2} ; P_{z}\right)$ at $\left|\vec{\Delta}_{T}\right|=0$ and 0.5 , respectively

the $x-k_{T}$ regions below straight line $y=-0.25 x+0.2$ are related to positive $F_{1}^{o}\left(x, \xi, \vec{k}_{T}^{2}, \vec{\Delta}_{T}^{2}\right)$. Then we compare the two upper panels in Fig. 1 and find that the $F_{1}^{o}\left(x, \xi, \vec{k}_{T}^{2}, \vec{\Delta}_{T}^{2}\right)$ result in the right panel is larger than that in the left panel at the same $x-k_{T}$ point. Comparing the upper panels with the lower panels, we emphasize that the two corresponding contours at $x>0.5$ have nearly the same shape.

Figure 2 depicts the T-odd GTMD $\tilde{G}_{1}^{o}\left(x, \xi, \vec{k}_{T}^{2}, \vec{\Delta}_{T}^{2}\right)$ as functions of $x$ and $\vec{k}_{T}$, where the paremeter values of contours are the same as Fig. 1. In two left panels, the $\tilde{G}_{1}^{o}\left(x, \xi, \vec{k}_{T}^{2}, \vec{\Delta}_{T}^{2}\right)$ result increases as $x$ or $k_{T}$ increases. Comparing two left panels, we find that the $\tilde{G}_{1}^{o}\left(x, \xi, \vec{k}_{T}^{2}, \vec{\Delta}_{T}^{2}\right)$ result in the lower panel is slightly smaller than that in the upper panel at the same $x-k_{T}$ point; While the $\tilde{G}_{1}^{o}\left(x, \xi, \vec{k}_{T}^{2}, \vec{\Delta}_{T}^{2}\right)$ results in two right panels are usually positive. Unlike two right panels in Fig. 1 where we plot contours at $\left|\vec{\Delta}_{T}\right|=0.5$, we show the result at $\left|\vec{\Delta}_{T}\right|=0.1$. Such difference indicates that the allowable maximum of $\left|\vec{\Delta}_{T}\right|$ for reaching nonvanishing T-odd GTMD $\tilde{G}_{1}^{o}\left(x, \xi, \vec{k}_{T}^{2}, \vec{\Delta}_{T}^{2}\right)$ becomes smaller in terms of that for $F_{1}^{o}\left(x, \xi, \vec{k}_{T}^{2}, \vec{\Delta}_{T}^{2}\right)$. We plot the Todd GTMD $H_{1}^{k, o}\left(x, \xi, \vec{k}_{T}^{2}, \vec{\Delta}_{T}^{2}\right)$ as functions of $x$ and $\vec{k}_{T}$ in
Fig. 3, where the paremeter values of contours are the same as Fig. 1. All four panels basically show the same shape of contours. Moreover, the maximum absolute value of the Todd GTMD $H_{1}^{k, o}\left(x, \xi, \vec{k}_{T}^{2}, \vec{\Delta}_{T}^{2}\right)$ can achieve 0.56 which is a larger value than those in Figs. 1 and 2. In Fig. 4, the Todd GTMD $H_{1}^{\Delta, o}\left(x, \xi, \vec{k}_{T}^{2}, \vec{\Delta}_{T}^{2}\right)$ as a function of $x$ and $\vec{k}_{T}$ at $\left|\vec{\Delta}_{T}\right|=0.1$ has been shown. Note that this GTMD becomes zero when $\left|\vec{\Delta}_{T}\right|=0$.

In the following we turn to the results of quasi-TMD $h_{1 \pi, Q(3)}^{\perp}$ and quasi-GPD $H_{1, Q(3)}$. In Fig. 5, we plot the quasi$\operatorname{TMD} h_{1 \pi, Q(3)}^{\perp}\left(x, \vec{k}_{T}^{2} ; P_{z}\right)$ as functions of $x$ and $k_{T}$ at different values of $P_{z}$ and TMD $h_{1 \pi}^{\perp}\left(x, \vec{k}_{T}^{2}\right)$ as functions of $x$ and $k_{T}$. We can see from the upper left panel that the absolute value of quasi-TMD $h_{1 \pi, Q(3)}^{\perp}\left(x, \vec{k}_{T}^{2} ; P_{z}\right)$ becomes larger as $k_{T}$ close to zero. In some region with negative $x$, the corresponding $h_{1 \pi}^{\perp}\left(x, \vec{k}_{T}^{2}\right)$ has nonvanishing values. As $x$ increases from -0.3 to 1 , the resulting $h_{1 \pi}^{\perp}\left(x, \vec{k}_{T}^{2}\right)$ absolute value gets larger first and then becomes smaller. For a nonvanishing $h_{1 \pi}^{\perp}\left(x, \vec{k}_{T}^{2}\right)$ result, the allowable maximum value of $k_{T}$ is around 0.38. Comparing the three panels in Fig. 5 depicting the quasi-TMD $h_{1 \pi, Q(3)}^{\perp}\left(x, \vec{k}_{T}^{2} ; P_{z}\right)$ with $P_{z}=1,3,5 \mathrm{GeV}$, 
we can find that as the $P_{z}$ value increases, the minimum of $x$ inside the contours becomes larger. In the lower left panel with $P_{z}=5 \mathrm{GeV}$, the quasi-TMD $h_{1 \pi, Q(3)}^{\perp}\left(x, \vec{k}_{T}^{2} ; P_{z}\right)$ result stay the same at a fixed $k_{T}$ value except the case $x$ close to 0 or 1 . The TMD $h_{1 \pi}^{\perp}\left(x, \vec{k}_{T}^{2}\right)$ as a negative function of $x$ and $k_{T}$ is shown in the lower right panel of Fig. 5. The absolute value of $h_{1 \pi}^{\perp}\left(x, \vec{k}_{T}^{2}\right)$ can reach 0.28 in almost all the $x$ range at a very small value of $k_{T}$.

Finally, we display the quasi-GPD $H_{1, Q(3)}\left(x, \xi, \vec{\Delta}_{T}^{2} ; P_{z}\right)$ as functions of $x$ and $P_{z}$ for different $\xi$ and $\left|\vec{\Delta}_{T}\right|$ values in Fig. 6. This quasi-GPD is negative. In the upper left panel, it is desired to mention that when $P_{z}>3 \mathrm{GeV}$, the quasi-GPD $H_{1, Q(3)}\left(x, \xi, \vec{\Delta}_{T}^{2} ; P_{z}\right)$ result hardly depends on the value of $P_{z}$. After comparing the two upper panels, we can acquire that the allowable range of $x$ for the nonvanishing quasi-GPD $H_{1, Q(3)}\left(x, \xi, \vec{\Delta}_{T}^{2} ; P_{z}\right)$ becomes larger when $\left|\vec{\Delta}_{T}\right|$ slightly increases from zero. Two panels have the very similar contour shape. When $\xi=0.5$, the quasi-GPD $H_{1, Q(3)}\left(x, \xi, \vec{\Delta}_{T}^{2} ; P_{z}\right)$ result is shown in two lower panels of Fig. 6. The absolute values of $H_{1, Q(3)}\left(x, \xi, \vec{\Delta}_{T}^{2} ; P_{z}\right)$ in two lower panels are smaller than those in two upper panels. We also find that when $P_{z}>3 \mathrm{GeV}$, the quasi-GPD $H_{1, Q(3)}\left(x, \xi, \vec{\Delta}_{T}^{2} ; P_{z}\right)$ result hardly depends on the value of $P_{z}$.

\section{Conclusion}

In this paper we have computed four T-odd GTMDs, quasiTMD and quasi-GPD in a scalar spectator model. We have present the results for four T-odd GTMDs. To get nonzero results for these functions requires considering at least oneloop corrections that include effects from the Wilson line. We have studied the relation of GTMDs for different values of skewness $\xi$ defined as the longitudinal momentum transferred to the proton and the total momentum transferred to the proton $\left|\vec{\Delta}_{T}\right|$. We found only in $x>\xi$ region, the T-odd GTMDs has nonvanishing value. Generally, the four T-odd GTMDs are negative in $x-k_{T}$ space. However, the $\tilde{G}_{1}^{o}\left(x, \xi, \vec{k}_{T}^{2}, \vec{\Delta}_{T}^{2}\right)$ results in certain parameter space are positive. Note that the T-odd GTMD $H_{1}^{\Delta, o}\left(x, \xi, \vec{k}_{T}^{2}, \vec{\Delta}_{T}^{2}\right)$ becomes zero when $\left|\vec{\Delta}_{T}\right|=0$. We have also considered the distributions of quasi-TMD and quasi-GPD. For the contours of quasi-TMD, we can find that as the $P_{z}$ value increases, the minimum of $x$ inside the contours becomes larger. We also find that when $P_{z}>3 \mathrm{GeV}$, the quasi-GPD $H_{1, Q(3)}\left(x, \xi, \vec{\Delta}_{T}^{2} ; P_{z}\right)$ result hardly depends on the value of $P_{z}$. The model calculations of the T-odd GTMDs, and Todd pion TMD - the Boer Mulders function can provide both reliable quantitative estimates and qualitative intuition that can be a valuable guide informing first principle calculations. Our study could prompt to further investigate the momentum structure, both collinear and three-dimension, of the pion especially given the paucity of work on the pion as compared to the nucleon on the topic of quasi PDFs and GPDs.

Acknowledgements Hao Sun is supported by the National Natural Science Foundation of China (Grant no. 11675033) and by the Fundamental Research Funds for the Central Universities (Grant no. DUT18LK27).

Data Availability Statement This manuscript has no associated data or the data will not be deposited. [Authors' comment: The datasets generated during and analysed during the current study are available from the corresponding author on reasonable request.]

Open Access This article is licensed under a Creative Commons Attribution 4.0 International License, which permits use, sharing, adaptation, distribution and reproduction in any medium or format, as long as you give appropriate credit to the original author(s) and the source, provide a link to the Creative Commons licence, and indicate if changes were made. The images or other third party material in this article are included in the article's Creative Commons licence, unless indicated otherwise in a credit line to the material. If material is not included in the article's Creative Commons licence and your intended use is not permitted by statutory regulation or exceeds the permitted use, you will need to obtain permission directly from the copyright holder. To view a copy of this licence, visit http://creativecomm ons.org/licenses/by/4.0/.

Funded by $\mathrm{SCOAP}^{3}$.

\section{References}

1. M. Diehl, Phys. Rep. 388, 41 (2003). arXiv:hep-ph/0307382

2. A.V. Belitsky, A.V. Radyushkin, Phys. Rep. 418, 1 (2005). arXiv:hep-ph/0504030

3. M. Garcon, Eur. Phys. J. A 18, 389 (2003). arXiv:hep-ph/0210068

4. K. Goeke, M.V. Polyakov, M. Vanderhaeghen, Prog. Part. Nucl. Phys. 47, 401 (2001). arXiv:hep-ph/0106012

5. J.C. Collins, D.E. Soper, Nucl. Phys. B 194, 445 (1982)

6. A. Bacchetta, M. Diehl, K. Goeke, A. Metz, P.J. Mulders, M. Schlegel, JHEP 02, 093 (2007). arXiv:hep-ph/0611265

7. S. Meissner, A. Metz, K. Goeke, Phys. Rev. D 76, 034002 (2007). arXiv:hep-ph/0703176

8. S. Meissner, A. Metz, M. Schlegel, K. Goeke, JHEP 08, 038 (2008). arXiv:0805.3165

9. S. Meissner, A. Metz, M. Schlegel, JHEP 08, 056 (2009). arXiv:0906.5323

10. C. Lorcé, B. Pasquini, JHEP 09, 138 (2013). arXiv:1307.4497

11. S.J. Brodsky, D.S. Hwang, I. Schmidt, Phys. Lett. B 530, 99 (2002). arXiv:hep-ph/0201296

12. D. Dolgov et al., LHPC, TXL, Phys. Rev. D 66, 034506 (2002). arXiv:hep-lat/0201021

13. M. Gockeler, R. Horsley, D. Pleiter, P. E. Rakow, and G. Schierholz, (QCDSF), Phys. Rev. D 71, 114511 (2005). arXiv:hep-ph/0410187

14. X. Ji, Phys. Rev. Lett. 110, 262002 (2013). arXiv:1305.1539

15. Y.-Q. Ma, J.-W. Qiu, Phys. Rev. D 98, 074021 (2018). arXiv: 1404.6860

16. X. Ji, Sci. China Phys. Mech. Astron. 57, 1407 (2014). arXiv: 1404.6680

17. H.-W. Lin, J.-W. Chen, S.D. Cohen, X. Ji, Phys. Rev. D 91, 054510 (2015). arXiv:1402.1462

18. X. Ji, Y.-S. Liu, Y. Liu, J.-H. Zhang, Y. Zhao (2020), arXiv:2004.03543

19. K. Orginos, A. Radyushkin, J. Karpie, S. Zafeiropoulos, Phys. Rev. D 96, 094503 (2017). arXiv:1706.05373 
20. J. Green, K. Jansen, F. Steffens, Phys. Rev. Lett. 121, 022004 (2018). arXiv: 1707.07152

21. C. Alexandrou, K. Cichy, V. Drach, E. Garcia-Ramos, K. Hadjiyiannakou, K. Jansen, F. Steffens, C. Wiese, Phys. Rev. D 92, 014502 (2015). arXiv:1504.07455

22. J.-W. Chen, S.D. Cohen, X. Ji, H.-W. Lin, J.-H. Zhang, Nucl. Phys. B 911, 246 (2016). arXiv: 1603.06664

23. C. Alexandrou, K. Cichy, M. Constantinou, K. Hadjiyiannakou, K. Jansen, F. Steffens, C. Wiese, Phys. Rev. D 96, 014513 (2017a). arXiv: 1610.03689

24. J.-H. Zhang, J.-W. Chen, X. Ji, L. Jin, H.-W. Lin, Phys. Rev. D 95, 094514 (2017). arXiv:1702.00008

25. H.-W. Lin, J.-W. Chen, T. Ishikawa, J.-H. Zhang, (LP3), Phys. Rev. D 98, 054504 (2018). arXiv: 1708.05301

26. G.S. Bali et al., Eur. Phys. J. C 78, 217 (2018). arXiv:1709.04325

27. C. Alexandrou, S. Bacchio, K. Cichy, M. Constantinou, K. Hadjiyiannakou, K. Jansen, G. Koutsou, A. Scapellato, F. Steffens, EPJ Web Conf. 175, 14008 (2018a). arXiv: 1710.06408

28. J.-H. Zhang, L. Jin, H.-W. Lin, A. Schäfer, P. Sun, Y.-B. Yang, R. Zhang, Y. Zhao, and J.-W. Chen, (LP3), Nucl. Phys. B 939, 429 (2019). arXiv: 1712.10025

29. C. Alexandrou, K. Cichy, M. Constantinou, K. Jansen, A. Scapellato, F. Steffens, Phys. Rev. Lett. 121, 112001 (2018b). arXiv: 1803.02685

30. J.-W. Chen, L. Jin, H.-W. Lin, Y.-S. Liu, Y.-B. Yang, J.-H. Zhang, Y. Zhao, (2018). arXiv:1803.04393

31. J.-H. Zhang, J.-W. Chen, L. Jin, H.-W. Lin, A. Schäfer, Y. Zhao, Phys. Rev. D 100, 034505 (2019b). arXiv:1804.01483

32. C. Alexandrou, K. Cichy, M. Constantinou, K. Jansen, A. Scapellato, F. Steffens, Phys. Rev. D 98, 091503 (2018c). arXiv: 1807.00232

33. Y.-S. Liu et al., (Lattice Parton), Phys. Rev. D 101, 034020 (2020). arXiv: 1807.06566

34. H.-W. Lin, J.-W. Chen, X. Ji, L. Jin, R. Li, Y.-S. Liu, Y.-B. Yang, J.-H. Zhang, Y. Zhao, Phys. Rev. Lett. 121, 242003 (2018b). arXiv: 1807.07431

35. L. Gamberg, Z.-B. Kang, I. Vitev, H. Xing, Phys. Lett. B 743, 112 (2015). arXiv:1412.3401

36. A. Bacchetta, M. Radici, B. Pasquini, X. Xiong, Phys. Rev. D 95, 014036 (2017). arXiv: 1608.07638

37. S.-I. Nam, Mod. Phys. Lett. A 32, 1750218 (2017). arXiv: 1704.03824

38. W. Broniowski, E. Ruiz Arriola, Phys. Lett. B 773, 385 (2017). arXiv: 1707.09588

39. T. Hobbs, Phys. Rev. D 97, 054028 (2018). arXiv:1708.05463

40. W. Broniowski, E. Ruiz Arriola, Phys. Rev. D 97, 034031 (2018). arXiv: 1711.03377

41. S.-S. Xu, L. Chang, C.D. Roberts, H.-S. Zong, Phys. Rev. D 97, 094014 (2018). arXiv:1802.09552

42. J.-W. Chen, T. Ishikawa, L. Jin, H.-W. Lin, Y.-B. Yang, J.-H. Zhang, Y. Zhao, Phys. Rev. D 97, 014505 (2018b). arXiv:1706.01295

43. X. Ji, J.-H. Zhang, Y. Zhao, Phys. Rev. Lett. 120, 112001 (2018). arXiv: 1706.08962

44. T. Ishikawa, Y.-Q. Ma, J.-W. Qiu, S. Yoshida, Phys. Rev. D 96, 094019 (2017). arXiv:1707.03107

45. C. Alexandrou, K. Cichy, M. Constantinou, K. Hadjiyiannakou, K. Jansen, H. Panagopoulos, F. Steffens, Nucl. Phys. B 923, 394 (2017b). arXiv:1706.00265

46. M. Constantinou, H. Panagopoulos, Phys. Rev. D 96, 054506 (2017). arXiv:1705.11193
47. X. Xiong, T. Luu, U.-G. Meißner (2017), arXiv:1705.00246

48. J.-W. Chen, X. Ji, J.-H. Zhang, Nucl. Phys. B 915, 1 (2017). arXiv: 1609.08102

49. T. Ishikawa, Y.-Q. Ma, J.-W. Qiu, S. Yoshida, (2016). arXiv: 1609.02018

50. X. Ji, P. Sun, X. Xiong, F. Yuan, Phys. Rev. D 91, 074009 (2015). arXiv: 1405.7640

51. X. Ji, L.-C. Jin, F. Yuan, J.-H. Zhang, Y. Zhao, Phys. Rev. D 99, 114006 (2019a). arXiv:1801.05930

52. M.A. Ebert, I.W. Stewart, Y. Zhao, Phys. Rev. D 99, 034505 (2019a). arXiv:1811.00026

53. M.A. Ebert, I.W. Stewart, Y. Zhao, JHEP 09, 037 (2019b). arXiv: 1901.03685

54. M.A. Ebert, I.W. Stewart, Y. Zhao, JHEP 03, 099 (2020a). arXiv: 1910.08569

55. X. Ji, Y. Liu, Y.-S. Liu, (2019). arXiv:1910.11415

56. X. Ji, Y. Liu, Y.-S. Liu, (2019). arXiv:1911.03840

57. A. A. Vladimirov, A. Sch äfer, Phys. Rev. D 101, 074517 (2020). arXiv:2002.07527

58. M.A. Ebert, S.T. Schindler, I.W. Stewart, Y. Zhao, (2020). arXiv:2004.14831

59. P. Shanahan, M.L. Wagman, Y. Zhao, Phys. Rev. D 101, 074505 (2020a). arXiv:1911.00800

60. P. Shanahan, M. Wagman, Y. Zhao, (2020). arXiv:2003.06063

61. S. Bhattacharya, C. Cocuzza, A. Metz, Phys. Lett. B 788, 453 (2019a). arXiv:1808.01437

62. S. Bhattacharya, C. Cocuzza, A. Metz, (2019). arXiv:1903.05721

63. Z.-L. Ma, J.-Q. Zhu, Z. Lu, (2019). arXiv:1912.12816

64. W. Broniowski, E. Ruiz Arriola, Phys. Lett. B 574, 57 (2003). arXiv:hep-ph/0307198

65. A.E. Dorokhov, W. Broniowski, E. Ruiz Arriola, Phys. Rev. D 84, 074015 (2011). arXiv: 1107.5631

66. R.M. Davidson, E. Ruiz Arriola, Acta Phys. Polon. B 33, 1791 (2002). arXiv:hep-ph/0110291

67. L. Theussl, S. Noguera, V. Vento, Eur. Phys. J. A 20, 483 (2004). arXiv:nucl-th/0211036

68. T. Frederico, E. Pace, B. Pasquini, G. Salme, Phys. Rev. D 80, 054021 (2009). arXiv:0907.5566

69. D. Brömmel et al., QCDSF, UKQCD, Phys. Rev. Lett. 101, 122001 (2008). arXiv:0708.2249

70. R. S. Sufian, C. Egerer, J. Karpie, R. G. Edwards, B. Joó, Y.-Q. Ma, K. Orginos, J.-W. Qiu, D. G. Richards, (2020). arXiv:2001.04960

71. T. Izubuchi, L. Jin, C. Kallidonis, N. Karthik, S. Mukherjee, P. Petreczky, C. Shugert, S. Syritsyn, Phys. Rev. D 100, 034516 (2019). arXiv: 1905.06349

72. J.-W. Chen, H.-W. Lin, J.-H. Zhang, (2019). arXiv:1904.12376

73. V. Barone, F. Bradamante, A. Martin, Prog. Part. Nucl. Phys. 65, 267 (2010). arXiv:1011.0909

74. D. Boer, P. Mulders, Phys. Rev. D 57, 5780 (1998). arXiv:hep-ph/9711485

75. A. Radyushkin, Phys. Lett. B 767, 314 (2017). arXiv: 1612.05170

76. M. Gluck, E. Reya, A. Vogt, Z. Phys. C 53, 651 (1992)

77. Z. Lu, B.-Q. Ma, Phys. Rev. D 70, 094044 (2004). arXiv:hep-ph/0411043

78. M. Burkardt, B. Hannafious, Phys. Lett. B 658, 130 (2008). arXiv:0705.1573 